\title{
Community paramedicine: A systematic review of program descriptions and training
}

\author{
Joyce Chan, B.Arts Sc, $\mathrm{MPH}^{+}$; Lauren E. Griffith, BS, MS, PhD'; Andrew P. Costa, BSc, PhD ;
} Matthew S. Leyenaar, BSc, $\mathrm{MA}^{\dagger}$; Gina Agarwal, MBBS(UK), MRCGP(UK) ${ }^{*}$

\section{CLINICIAN'S CAPSULE \\ What is known about the topic? \\ Despite a growing global interest in community parame- dicine, there is poor understanding of the program types and training for each. \\ What did this study ask? \\ What are the different types of community paramedicine programs and the training for each type? \\ What did this study find? \\ Community paramedicine programs were diverse and collaborative, often serving 911 callers and in-home visits, and training emphasized technical skills. \\ Why does this study matter to clinicians? \\ Understanding programs and training informs areas of interest, including for emergency medicine providers, such as programming, education, and regulation.}

\section{ABSTRACT}

Objectives: The aim of this study is to identify the types of community paramedicine programs and the training for each.

Methods: A systematic review of MEDLINE, Embase, grey literature, and bibliographies followed a search strategy using common community paramedicine terms. All studies published in English up to January 22, 2018, were captured. Screening and extraction were completed in duplicate by two independent reviewers. The Mixed Methods Appraisal Tool (MMAT) was used to assess studies' methodological quality (full methodology on PROSPERO: CRD42017051774).

Results: From 3,004 papers, there were 64 papers identified (58 unique community paramedicine programs). Of the papers with an appraisable study design $(40.6 \%)$, the median MMAT score was 3 of 4 criteria met, suggesting moderate quality. Programs most often served frequent 911 callers (48.3\%) and individuals at risk for emergency department admission, readmission, or hospitalization (41.4\%); and $70.7 \%$ of programs were preventive home visits. Common services provided were home assessment
(29.5\%), medication management $(39.7 \%)$, and referral and/or transport to community services (37.9\%); and $77.6 \%$ of programs involved interprofessional collaboration. Community paramedicine training was described by $57 \%$ of programs and expanded upon traditional paramedicine training and emphasized technical skills. Study heterogeneity prevented meta-analysis.

Conclusion: Community paramedicine programs and training were diverse and allowed community paramedics to address a spectrum of population health and social needs. Training was poorly described. Enabling more programs to assess and report on program and training outcomes would support community paramedicine growth and the development of formalized training or education frameworks.

\section{RÉSUMÉ}

Objectif: L'étude visait à relever les différents types de programmes de paramédecine communautaire et à décrire la formation donnée dans chacun d'eux.

Méthode: Une revue systématique des bases de données MEDLINE et Embase, de la documentation parallèle ainsi que de bibliographies a été entreprise à la suite d'une stratégie de recherche élaborée à l'aide de termes utilisés souvent en paramédecine communautaire. Ont été saisies toutes les études publiées en anglais jusqu'au 22 janvier 2018. Le tri et l'extraction des données ont été faits en double, par deux examinateurs indépendants. L'évaluation de la qualité méthodologique des études a été réalisée à l'aide de l'instrument Mixed Methods Appraisal Tool (MMAT) (description complète de la méthode dans PROSPERO : CRD42017051774).

Résultats: Sur 3004 articles relevés, 64 ont été retenus (58 programmes distincts de paramédecine communautaire). Le score médian MMAT des articles présentant un plan d'étude susceptible d'évaluation $(40,6 \%)$ était de 3 sur 4 quant au respect des critères établis, résultat évocateur d'une qualité moyenne. Les programmes avaient surtout pour cible les usagers fréquents du service 911 (48,3\%) et les personnes susceptibles d'admission ou de réadmission au service des urgences, ou encore d'hospitalisation $(41,4 \%) ; 70,7 \%$ des programmes

From the Departments of *Family Medicine; and the HHealth Research Methods, Evidence, and Impact; McMaster University, Hamilton, ON.

Correspondence to: Dr. Gina Agarwal, McMaster University Family Medicine, David Braley Health Sciences Centre, 100 Main Street West, 6th Floor, Hamilton, ON L8P 1H6; Email: gina.agarwal@gmail.com

(C) Canadian Association of Emergency Physicians 
portaient sur les visites préventives à domicile. Les services fréquemment offerts étaient les évaluations à domicile $(29,5 \%)$, le contrôle de la pharmacothérapie $(39,7 \%)$ et l'orientation ou le transport des malades vers des services communautaires (37,9\%); 77,6\% des programmes incluaient un volet de collaboration interprofessionnelle. La formation en paramédecine communautaire a été décrite par $57 \%$ des programmes et étendu sur le champ de pratique habituel de la paramédecine traditionnelle et visait l'acquisition de compétences techniques. Enfin, il n'a pas été possible de procéder à une méta-analyse en raison de l'hétérogénéité des études.

Conclusion: Les programmes de paramédecine communautaire et la formation afférente sont diversifiés et permettent, de ce fait, aux professionnels du domaine de répondre à un large éventail de besoins sociaux et de besoins en matière de santé de la population. Pour ce qui est des descriptions de la formation donnée, elles étaient insuffisantes. Si les responsables de programmes étaient tenus d'évaluer les programmes et la formation offerte et de faire état des résultats obtenus, cela favoriserait le développement de la paramédecine communautaire et l'élaboration de cadres structurés d'études ou de formation.

Keywords: Community paramedic, community paramedicine, emergency medical services, mobile integrated healthcare, paramedicine

\section{INTRODUCTION}

Community paramedicine is an emerging form of health services delivery with the potential to reduce emergency department (ED) visits among high user groups while making use of existing paramedic resources. ${ }^{1-3}$ There is growing interest in community paramedicine and its expansion across Canada, Australia, the United States, and the United Kingdom. ${ }^{4}$ Community paramedicine extends traditional paramedic care and is staffed by emergency medical services (EMS) professionals often with additional training. ${ }^{5}$ Programs can be tailored to community needs by providing a range of services, including disease management, home assessments, and referral to community services. ${ }^{6,7}$

Community paramedicine programs may lead to more effective use of paramedic resources. In some programs, paramedics on accommodated duty have adopted the community paramedic role while awaiting return to regular duties. ${ }^{6}$ Community paramedicine responsibilities have also been incorporated into the downtime between emergency calls. ${ }^{8}$ Community paramedicine may support more efficient use of healthcare resources by increasing collaboration between different healthcare providers $^{2}$ and facilitating patients' access to appropriate home and community services. ${ }^{6}$

Despite expanding upon the paramedicine role, many countries have no professional education standard for community paramedicine - Canada being one of them. Training for community paramedicine usually varies by program or region. In Ontario, the provincial government regulates the qualifications and roles of only paramedics providing ambulance services, not those of community paramedics. Furthermore, provincial legislation in Alberta, Saskatchewan, Nova Scotia, and New Brunswick has allowed paramedics to be a self-regulated profession. ${ }^{9,10}$ Identification of common services and roles across community paramedicine programs will inform future discussions on community paramedicine education standards and professional regulation. The Canadian Standards Association's (CSA) community paramedicine framework for program development, which details types of community paramedicine programs, is a start. ${ }^{11}$ Discussion on the future of community paramedicine in Canada is also informed by recommendations on paramedicine scope of practice and direction from leading organizations in the paramedicine sector, including the EMS Chiefs of Canada and the Canadian Organization of Paramedic Regulators. In 2006, the EMS Chiefs of Canada published The White Paper, which outlined the strategic direction for EMS in Canada, including a focus on interprofessional collaboration. ${ }^{12}$ The Canadian Organization of Paramedic Regulators aims to be a collective voice for paramedic regulators and to support the development of paramedicine in Canada. Key pillars in sector development identified by the organization include intersectoral collaboration and leveraging evidence to assess and further paramedics' professional competencies. ${ }^{13}$

Despite community paramedicine growth, the current community paramedicine landscape, types of programs and their purpose, and the training required for each program type remain poorly understood. ${ }^{14,15}$ This information may guide development and evaluation of new programs, facilitate resource pooling between jurisdictions, and support regional planning for community 
paramedicine programs. Knowledge of community paramedicine training required to support different types of programming may also inform the direction of the community paramedic profession. A systematic review was conducted to 1) identify the key differences between community paramedicine programs for program classification, and 2) describe the training required for each program type.

\section{METHODS}

\section{Study type}

A systematic review was conducted according to Cochrane methodology. The full methodology is available on PROSPERO (registration number: CRD42017051774) ${ }^{16}$ and is summarized below. Systematic review methods were chosen because the research questions were specific and well defined, and the included studies could be assessed for quality. ${ }^{17}$ In contrast, scoping reviews generally have broader research questions, and quality assessment of included studies is often not done. ${ }^{18}$

\section{Research questions}

1) What are the key differences between community paramedicine programs for program classification?

2) What is the training required for each type of community paramedicine program?

\section{Data sources and search strategy}

MEDLINE and Embase databases were searched to identify all relevant articles published up until January 22, 2018. Keywords from frequently cited community paramedicine articles were used with advice from experts and research librarians. The keywords reflect the most preliminary conceptualization of community paramedicine, that is, it involves paramedics providing services in a community setting. The strategy combined terms from three themes: 1) paramedicine and paramedics, 2) community setting, and 3) synonyms for community paramedicine such as $C P$ and Mobile Integrated Healthcare $(\mathrm{MIH})$. The MEDLINE search strategy [((theme 1 terms combined with OR) Emergency care practitioner.mp., Paramedic*.mp., Paramedical personnel.mp., Para medical personnel.mp.) AND ((theme 2 terms combined with OR) Community care.mp., Community.mp., Communities.mp.)] OR [(theme 3 terms combined with OR) Community paramedic*.mp., Mobile integrated bealthcare.mp., Mobile integrated bealth care.mp., MIH-CP.mp., Community paramedicine program*.mp.] was mirrored for Embase. Additional articles were identified by hand-searching bibliographies and grey literature searches for programs identified in included articles, and by community paramedicine experts.

MEDLINE and Embase databases were selected because, through preliminary searching of the literature, it seemed that most of the published literature would be captured in these databases. Recognizing that a lot of programs are described in the grey literature, additional hand-searching of bibliographies and grey literature searching were completed. Grey literature searching was done through a targeted Google search using the names of community paramedicine programs and any other identifying information (e.g., location of program) referenced in the screened articles. The types of grey literature materials included government and community reports, newspaper articles, and poster presentations.

\section{Inclusion criteria and selection process}

All articles describing community paramedicine programming were included regardless of study design, setting, population, or outcomes. Non-English articles were excluded. Title and abstract (Level 1) and full-text (Level 2) screening of the published articles were completed in duplicate by two independent reviewers (JC, GA). Grey literature sources were also screened in duplicate by two independent reviewers (JC, GA). Cohen's kappa coefficient ( $\kappa$ ) was used to measure inter-rater agreement. Data extraction for the published and grey literature sources was completed in duplicate by independent reviewers JC and GA. All discrepancies were resolved by discussion or consultation with a third reviewer (AC or LG).

Primary outcomes extracted for the published and grey literature sources were locations of community paramedicine visits (e.g., home visit), patient populations (e.g., 911 callers), target conditions addressed (e.g., diabetes), method of patient enrolment (e.g., referral from healthcare provider), interprofessional team members (i.e., who work with the community paramedic), community paramedicine services provided (e.g., acute care), health outcomes assessed (e.g., transport to ED), and community paramedicine training, including training subjects, provider, and duration. Excel spreadsheets 
were used to organize the screening and data extracted from studies identified in the published and grey literatures.

\section{Study quality assessment}

The 2011 version of the Mixed Methods Appraisal Tool (MMAT) was used to evaluate the methodological quality of included studies with one of five study designs (qualitative, quantitative randomized controlled, quantitative non-randomized, quantitative descriptive, and mixed methods). The MMAT has been used in over 50 systematic reviews worldwide and is reliable and content validated. ${ }^{19}$ Other study designs (e.g., literature reviews) and articles with no study design (e.g., only described the program) or results (e.g., protocol) could not be assessed by the MMAT.

\section{RESULTS}

\section{Part I. Search yield}

The search identified 3,004 articles, and, after screening and searching bibliographies of included articles, a total of 64 studies representing 58 unique community paramedicine programs were included (Figure 1)..$^{1,2,3,6,7,20-78}$

There were 15 (23.4\%) of the 64 studies identified by hand-searching the bibliographies of included studies and by follow-up grey literature searching, and 14 (24.1\%) of 58 community paramedicine programs captured were identified through the grey literature (see online Appendix for the table of included studies and grey literature sources).

Of the 64 studies, $29(45.3 \%)$ studies had a defined study design and $35(54.7 \%)$ described the program only. Of those with a study design that could be

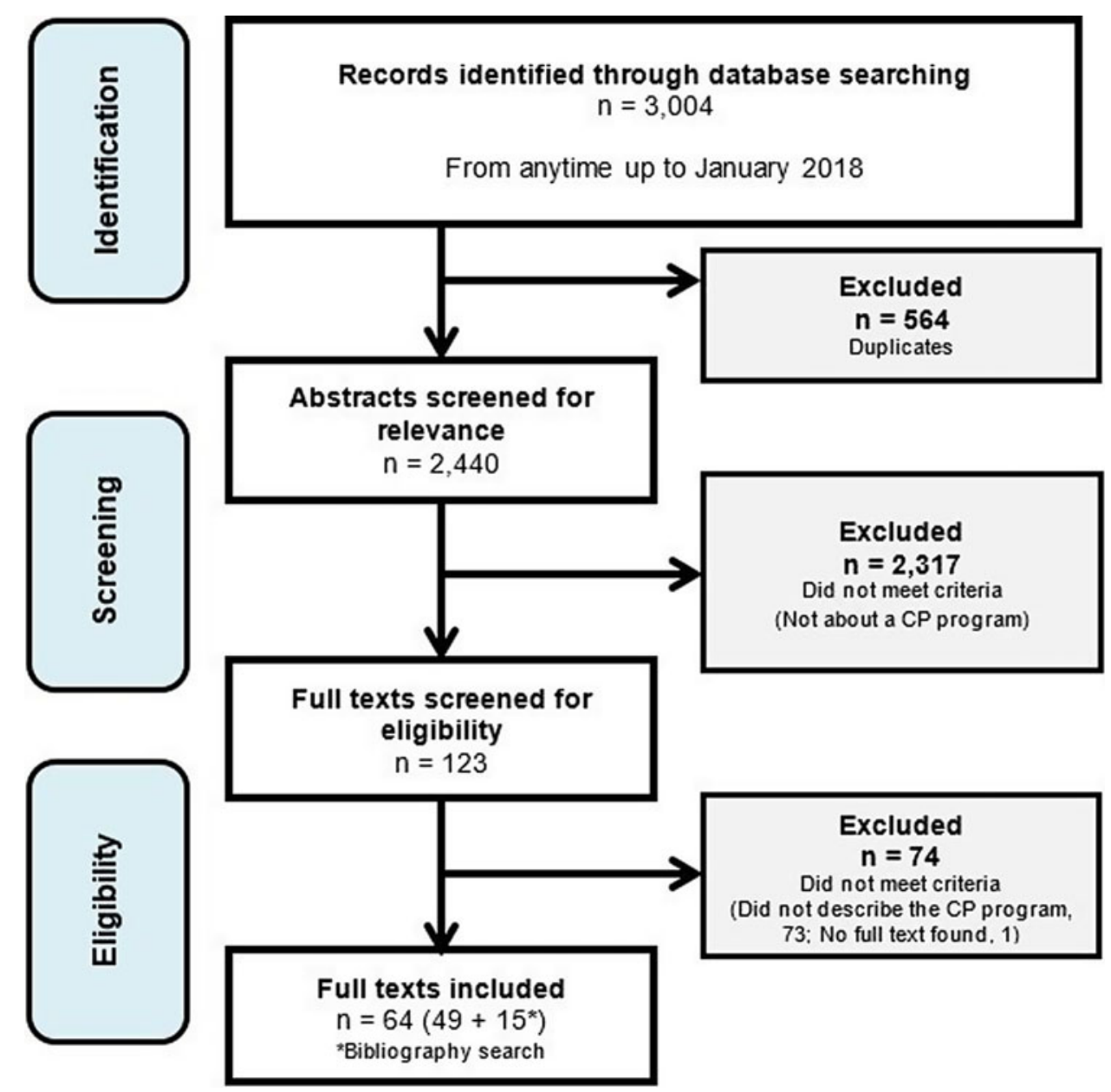

Figure 1. 
appraised using the MMAT, 6 were quantitative randomized controlled studies (e.g., randomized controlled trial [RCT]), 11 were quantitative non-randomized (e.g., case-control, cohort), 8 were quantitative descriptive studies (e.g., incidence/prevalence study without comparator), and 4 were mixed methods studies (e.g., cluster RCT with qualitative interviews). The MMAT was applied to the 26 studies ( 3 studies were protocol only); the median score was three stars, or three of four criteria met, suggesting moderate quality. Levels 1 and 2 screening were completed in duplicate. The raw agreement and kappa coefficient for L1 pilot $(\mathrm{n}=200)$ were $94 \%$ and 0.78 , respectively, and $95 \%$ and 0.42 for L1 total $(n=2,804)$. For L2 pilot $(n=11)$, they were $73 \%$ and 0.42 , respectively, and $100 \%$ and 1.00 for L2 total $(\mathrm{n}=112)$.

\section{Part II. Features of the 58 community paramedicine programs captured}

\section{Location of community paramedicine visits}

All 58 programs stated the community paramedicine visit location, with most operating through home visits only $(70.7 \%)$. There were $46(79.3 \%)$ urban area programs and $7(12.1 \%)$ in rural areas only (Table 1$)$.

\section{Patient populations}

The target population of $28(48.3 \%)$ programs was emergency callers (e.g., called 911 or were frequent

\begin{tabular}{lc|}
\hline Table 1. Location of community paramedicine visits* \\
\hline Place of visit & Number of programs ( $\mathrm{n}=58)$ \\
\hline Patient home & $41(70.7 \%)$ \\
Place of 911 call incidence & $10(17.2 \%)$ \\
Community clinic & $4(6.9 \%)$ \\
Common area in residence building & $2(3.4 \%)$ \\
Telephone services & $1(1.7 \%)$ \\
Hospice & $1(1.7 \%)$ \\
Hospital & $1(1.7 \%)$ \\
Long-term care (LTC) facility & $1(1.7 \%)$ \\
\hline Community type & Number of programs $(n=58)$ \\
\hline Urban & $46(79.3 \%)$ \\
Rural & $7(12.1 \%)$ \\
Urban and rural (e.g., multi-site & $1(1.7 \%)$ \\
$\quad$ community paramedicine & \\
program) & $4(6.9 \%)$ \\
\hline Unknown & \\
\hline *Categories are non-exclusive (i.e., a program can have more than one visit location).
\end{tabular}

911 callers), and there were 24 (41.4\%) programs for individuals at risk for ED admission or readmission, or hospitalization. Overall, seven $(12.1 \%)$ programs were solely for older adults living in the community or longterm care (LTC) homes (Table 2).

\section{Target conditions and patient enrolment}

The majority of community paramedicine programs $(\mathrm{n}=39 ; 67.2 \%)$ did not target a specific health condition. Among those that did, diabetes mellitus was common $(\mathrm{n}=6 ; 10.3 \%)$, followed by heart failure $(\mathrm{n}=5 ; 8.6 \%)$ and chronic obstructive pulmonary disease $(n=5$; 8.6\%). An emergency call initiated patient enrolment in $22(37.9 \%)$ programs; in $11(19.0 \%)$ programs, clients were referred (e.g., by a provider, social worker, family); and clients voluntarily enrolled in $6(10.3 \%)$ after receiving an invitation or hearing about the program.

\section{Interprofessional collaboration}

In 45 (77.6\%) programs, community paramedics collaborated with at least one other professional: nurses, including nurse practitioners $(\mathrm{n}=11 ; 19.0 \%)$, family doctors only $(\mathrm{n}=3 ; 5.2 \%)$, primary care teams, which

\begin{tabular}{lc|}
\hline Table 2. Patient populations* & $\begin{array}{c}\text { Number of programs } \\
(\mathrm{n}=58)\end{array}$ \\
\hline $\begin{array}{l}\text { Patient population } \\
\text { - In general }\end{array}$ & $28(48.3 \%)$ \\
& $3(5.2 \%)$ \\
- Presenting with low acuity conditions & $12(20.7 \%)$ \\
- Presenting with low acuity conditions & $3(5.2 \%)$ \\
and are seniors in the community or LTC & \\
home & \\
- Frequent 911 callers/EMS users & $10(17.2 \%)$ \\
At risk for ED readmission or hospitalization & $24(41.4 \%)$ \\
- In general & $22(37.9 \%)$ \\
- Children & $1(1.7 \%)$ \\
- Hospice patients & $1(1.7 \%)$ \\
$\begin{array}{l}\text { Seniors living in the community (not facility) } \\
\text { Other (e.g., families with newborns) } \\
\text { Unknown }\end{array}$ & $4(6.9 \%)$ \\
\hline Categories are non-exclusive. & $1(1.7 \%)$ \\
\hline
\end{tabular}


may have included family doctors $(n=4 ; 6.9 \%)$, pharmacists $(n=4 ; 6.9 \%)$, and social workers $(n=7 ; 12.1 \%)$.

\section{Services provided}

The types of services provided included assessment and screening, acute care and treatment, transport and referral, education and patient support, communication, and other (Table 3). Most common were physical assessment $(\mathrm{n}=27 ; 46.6 \%)$, medication management $(\mathrm{n}=23$; $39.7 \%)$, and assessment, referral, and/or transport to community services $(n=22 ; 37.9 \%)$.

\section{Health outcomes assessed}

Health outcomes were extracted per study instead of per community paramedicine program because different studies of the same program evaluated different health outcomes. Of the 64 studies, 13 (20.3\%) did not report any health outcomes. The most commonly reported health outcomes included transport to $\mathrm{ED}(\mathrm{n}=23$; $35.9 \%)$, hospital admission $(\mathrm{n}=21 ; 32.8 \%)$, and 911 calls $(\mathrm{n}=12 ; 18.8 \%)$. Other patient outcomes assessed were adverse outcomes $(n=7 ; 10.9 \%)$, clinical improvements or changes $(n=10 ; 15.6 \%)$, healthcare utilization (non-ED, $\mathrm{n}=8 ; 12.5 \%)$, medication adherence $(\mathrm{n}=3$; $4.7 \%)$, non-healthcare resources utilization $(\mathrm{n}=3$; $4.7 \%)$, patient satisfaction $(\mathrm{n}=11 ; 17.2 \%)$, and treated on-scene $(n=4 ; 6.3 \%)$.

\section{Part III. Features of community paramedicine training: Subjects, providers, and duration}

Training components of the 58 community paramedicine programs were acute care, assessment and screening, care of specific populations, education and health promotion, special knowledge, as well as communication and leadership. Training components were not described by 25 programs (43.1\%, Table 4). There were $25(43.1 \%)$ programs that described the training provider(s), where $6(10.3 \%)$ involved a university (e.g., school of medicine), 9 (15.5\%) involved healthcare professionals (e.g., care of elderly specialists), and 4 (6.9\%) involved a college (e.g., technical college). Community services and representatives, hospitals, and public health departments were involved in two programs (3.4\%) each. Only $22(37.9 \%)$ community paramedicine programs provided clear information about the training duration; the median training time was 240 hours per trainee per program with a range of 3.5 to 2,080 hours.

\section{Part IV. Additional analyses: Community paramedicine services and training subjects by population}

Among the 28 community paramedicine programs that targeted 911 callers, the most common services provided were physical assessments $(\mathrm{n}=15 ; 53.6 \%)$, acute care $(\mathrm{n}=12 ; 42.9 \%)$, and transport to ED or urgent care centres $(\mathrm{n}=10 ; 35.7 \%)$. The most common training subjects for these 28 programs were acute care $(n=7 ; 25.0 \%)$, intervention-specific materials $(\mathrm{n}=7 ; 25.0 \%)$, health management $(n=6 ; 21.4 \%)$, comprehensive health assessment $(\mathrm{n}=6 ; 21.4 \%)$, and knowledge of community services $(\mathrm{n}=5 ; 17.9 \%)$.

Among the 24 community paramedicine programs for individuals at risk for ED admission, readmission or hospitalization, the most common services were medication management $(\mathrm{n}=14 ; 58.3 \%)$, followed by physical assessment, acute care, education, and communicating with the patient's healthcare provider(s) in 9 (37.5\%) programs each. For these 24 programs, the most common training subjects were health management $(n=8$; $33.3 \%)$, providing acute care $(n=6 ; 25.0 \%)$, medication management $(n=6 ; 25.0 \%)$, knowledge of community services $(\mathrm{n}=5 ; 20.8 \%)$, and how to care for seniors ( $\mathrm{n}=5 ; 20.8 \%$ ).

Among the four community paramedicine programs for seniors living in the community (not facility), the most common services provided were physical assessments and assessing, referring, and/or transporting to community services with each provided by three $(75.0 \%)$ programs. The training for these programs usually covered how to care for seniors; assessing the environment (e.g., home), health risks, and overall health; health promotion; and intervention-specific materials $(\mathrm{n}=1$ each; $25.0 \%)$.

\section{DISCUSSION}

In this comprehensive systematic review, the 58 community paramedicine programs evaluated were very heterogeneous in the populations they served and their outcomes captured. The community paramedicine training required to implement these programs was often sparsely described, if at all. Among the handful of existing literature reviews about community paramedicine,${ }^{5,14,15}$ none described the key differences between community paramedicine programs and the training for each. The present systematic review captured many 
Table 3. Services provided*

$\begin{array}{ll} & \text { Number of } \\ \text { Service type } & \text { programs }(n=58)\end{array}$

Assessment and screening

- Collect patient history information (e.g., medical history)

- Depression screening

- Assessment of the home

- Medication management (e.g., protocol led dispensing, medication review)

- Monitor patient (e.g., side effects/symptoms, mental health)

- Non-physical assessment (e.g., mental health and social needs assessments)

- Physical assessment (e.g., vital signs, blood pressure)

- Preventive health screening (e.g., not for depression)

Acute care and treatment

- Acute care (e.g., assess and treat minor issues/ conditions)

- Immunization

- Point-of-care lab tests (e.g., blood draws, toxicology screening)

Transport and referral

- Assess, refer, and/or transport to community services (e.g., sobering centre, detox centres, mental health crisis centre, mental health hospital)

- Refer and/or transport to additional healthcare providers (e.g., pharmacist, physician, hospital diagnostic imaging)

- Transport to ED/urgent care centre (e.g., walk-in clinic)

Education and patient support

- Care plan review (e.g., review discharge instructions; to ensure understanding, improve adherence)
$21(36.2 \%)$

$1(1.7 \%)$

$7(12.1 \%)$

$22(37.9 \%)$

$13(22.4 \%)$

$13(22.4 \%)$

$6(10.3 \%)$

(Continued) $3(5.2 \%)$

$1(1.7 \%)$

$14(29.5 \%)$

\section{Table 3. Continued.}

$\begin{array}{ll} & \text { Number of } \\ \text { Service type } & \text { programs }(n=58)\end{array}$

- Counselling (e.g., support for patient or family) 4 (6.9\%)

- Education (e.g., chronic disease management; 15 (25.9\%) navigating health system)

- Coaching (e.g., goal setting, personalized health coach)

- Phone consultation

Communication

- Communication with other healthcare $15(25.9 \%)$ providers (e.g., to co-determine care plans or relay information)

- Communicate with patient's family/caregivers $2(3.4 \%)$

$27(46.6 \%)$

Not reported

$4(6.9 \%)$ community paramedicine programs and described program training and services by population.

The diversity of community paramedicine roles and services has allowed community paramedics to address a variety of health and related community needs, as recommended by the CSA standards. ${ }^{11}$ Services associated with paramedicine, including acute care, physical assessment, and transport to the ED or urgent care centre, were part of the services and training for many community paramedicine programs captured. Commonalities between paramedicine and community paramedicine services may indicate that paramedics are in a good position to adopt the community paramedicine role because they already have some of the relevant skills. Community paramedicine expanded upon traditional paramedic duties, clientele, and interprofessional collaboration. Community paramedicine programs also provided education, counselling, coaching, health promotion activities, and in-home visits. In addition to serving 911 callers, commonly paramedicine clientele, many community paramedicine programs served individuals at risk for ED admission or readmission or hospitalization. Working with clients before their need for ED or hospital care demonstrates the unique role of community 


\begin{tabular}{|c|c|}
\hline Training & $\begin{array}{l}\text { Number of } \\
\text { programs }(n=58)\end{array}$ \\
\hline \multicolumn{2}{|l|}{ Acute care } \\
\hline - Acute care & $13(22.4 \%)$ \\
\hline - Diagnostic (e.g., point-of-care testing) & $5(8.6 \%)$ \\
\hline - Emergency care & $1(1.7 \%)$ \\
\hline \multicolumn{2}{|l|}{ Assessment and screening } \\
\hline - Environmental assessment & $3(5.2 \%)$ \\
\hline - Health risk assessment & $3(5.2 \%)$ \\
\hline $\begin{array}{l}\text { - Medication management (e.g., drug } \\
\text { interactions, medication review) }\end{array}$ & $10(17.2 \%)$ \\
\hline - Mental health assessment & $3(5.2 \%)$ \\
\hline $\begin{array}{l}\text { - Comprehensive health assessment } \\
\text { (physical, social, and mobility needs) }\end{array}$ & $8(13.8 \%)$ \\
\hline - Patient history & $2(3.4 \%)$ \\
\hline - Physical assessment & $1(1.7 \%)$ \\
\hline \multicolumn{2}{|l|}{ Care of specific populations } \\
\hline - Care of older adults & $9(20.5 \%)$ \\
\hline - Care of children & $4(6.9 \%)$ \\
\hline \multicolumn{2}{|l|}{ Education and health promotion } \\
\hline $\begin{array}{l}\text { - Assist health management (e.g., managing } \\
\text { chronic conditions, health coaching) }\end{array}$ & $13(22.4 \%)$ \\
\hline - Case management & $1(1.7 \%)$ \\
\hline - Education (e.g., self-care) & $5(8.6 \%)$ \\
\hline $\begin{array}{l}\text { - Health promotion (e.g., preventive care, } \\
\text { disease prevention) }\end{array}$ & $6(10.3 \%)$ \\
\hline \multicolumn{2}{|l|}{ Special knowledge } \\
\hline - Knowledge of community services & $10(17.2 \%)$ \\
\hline $\begin{array}{l}\text { - Intervention-specific materials and } \\
\text { procedures (e.g., video conferencing, } \\
\text { protocols) }\end{array}$ & $10(17.2 \%)$ \\
\hline
\end{tabular}

(Continued)

\begin{tabular}{lc|}
\hline Table 4. Continued. & $\begin{array}{c}\text { Number of } \\
\text { programs }(n=58)\end{array}$ \\
\hline Training & $1(1.7 \%)$ \\
\hline - Law enforcement & $1(1.7 \%)$ \\
- Substance abuse & \\
\hline $\begin{array}{l}\text { Communication and leadership } \\
\text { - Lommunication }\end{array}$ & $2(3.4 \%)$ \\
- Leadership & $1(1.7 \%)$ \\
\hline Not reported & $25(43.1 \%)$ \\
\hline${ }^{*}$ Categories are non-exclusive. & \\
\hline
\end{tabular}

paramedicine in providing preventive and upstream care. Diverse and expanding roles and services for paramedics, as observed in the community paramedicine programs captured, reflect a broader innovative approach by the paramedic sector to support community needs. The innovation and evolution of paramedicine models of care to better meet community needs align with the recommended direction for paramedicine in Canada developed by the EMS Chiefs of Canada. ${ }^{12}$

Only a handful of community paramedicine programs reported seniors as their sole target demographic and/or provided training on caring for senior populations. The number of community paramedicine programs serving seniors is likely underestimated because seniors are often present in other demographic groups targeted, including frequent 911 callers and ED users. For example, in a systematic review of ED use in the United States, frequent users were more likely to be between the ages of 25 and 44 or age 65 and up. ${ }^{79}$

The general understanding of community paramedicine as being paramedics with additional training in health prevention and promotion did not capture the observed spectrum of community paramedicine services and training. As demonstrated in this review, community paramedicine expands upon traditional paramedicine training and services, in that paramedics receive additional training to provide services that address immediate and anticipated health, social, and other needs - not just acute and emergent needs. This definition of community paramedicine aligns with the standard on community paramedicine program planning developed by the CSA in 2017 where community paramedicine 
programs were described as providing a spectrum of immediate and scheduled care to vulnerable patients to improve equity in healthcare access. ${ }^{11}$ There is also alignment with the International Roundtable on Community Paramedicine which viewed community paramedicine as the application of paramedicine skills and training, often with an expanded scope of practice (i.e., outside emergency transport and response), in community settings.

Adding to these definitions, community paramedicine is also shown here as a highly collaborative profession, because the majority of community paramedicine programs captured involved interprofessional collaboration and connected clients to other providers and community organizations. Interprofessional collaboration is not only a key characteristic of programs captured in the present review, but also, more broadly, may be contributing to overall improved community paramedicine program and sector outcomes. The EMS Chiefs of Canada propose that collaboration between EMS and community organizations (e.g., different healthcare providers and social services) enables the development of innovative initiatives that support improved healthcare in a community. ${ }^{12}$ Furthermore, the Canadian Organization of Paramedic Regulators identifies that collaboration enables the paramedicine sector to adapt more effectively to changes in scope of practice and regulation. ${ }^{13}$

The majority of community paramedicine training described in this review, however, seemed to be centred on technical skills such as acute care, assessment, and screening. The expanded activities, roles, and collaborations of community paramedics further underline the value of common and standardized community paramedicine training for relevant skills such as communication, teamwork, and leadership. Although standard paramedic training may already address skills such as communication, the diversity of community paramedicine services and roles emphasizes the value of more specialized community paramedicine training in the areas noted.

Despite this, the diversity of professional backgrounds captured among providers of community paramedicine training - such as public health, community services, and healthcare - may have contributed to community paramedics developing a more holistic understanding of community partners and services. Community paramedicine training may also have been a platform to clarify roles and interactions between community paramedics and other providers, and may have supported the day-to-day interprofessional collaboration observed in many community paramedicine programs. Role clarity has been shown to support optimized integration of providers into new healthcare settings and teams. ${ }^{80}$ Ensuring role clarity for community paramedics, such as scope of practice and responsibilities, within the interprofessional context may support integration of community paramedicine and effective interprofessional collaboration.

Among the programs that reported on training, most did not use regionally mandated standardized training, with the exception of programs based in Minnesota (United States) where community paramedicine certification followed a customizable state-wide curriculum. ${ }^{81}$ The overlap in subjects taught in community paramedicine training, especially among those programs serving the major community paramedicine target populations, may indicate core competencies for community paramedicine. The development of a common community paramedicine education framework could be informed by these core competencies.

A strength of community paramedicine programs was how roles and services were responsive to a variety of health and related community needs. The diversity of community paramedicine programs, however, has also made it challenging to develop a specific single role description for community paramedicine. As a result of community paramedicine serving diverse target populations and providing various services, community paramedicine training was often developed at the program-level and tailored to these factors, rather than standardized across programs. A standardized educational framework for community paramedicine would need to capture the core competencies required, but also allow for program-level customization to include a growing spectrum of community paramedicine competencies. The community paramedicine programs captured in this review often did not report training subjects and/or outcomes, and none described paramedics' training success and confidence in becoming a community paramedic. The limited knowledge of what was effective and ineffective for community paramedicine training is a considerable barrier to developing an evidence-informed community paramedicine education framework.

In order to facilitate discussion around standardized training or certification of the community paramedicine profession, there needs to be an adequate information "bank" about community paramedicine programs and training. The resources and capacity must be available 
for community paramedicine programs to undertake rigorous evaluation of program and training outcomes and to disseminate findings. There must also be expert input, such as from established community paramedicine programs, education institutions, professional bodies, the public, and other stakeholders, when determining the core components of community paramedicine training to inform any community paramedicine education framework.

In the United Kingdom, for example, the Health and Care Professions Standard (HCPS) regulates the paramedicine profession and has developed standards to regulate and approve education programs; HCPS has not developed comparable standards and regulations for community paramedicine. ${ }^{9}$ In the United States, many states have passed a legislation to enable and define community paramedicine practice, and determine education or licensing. ${ }^{82}$ The regulatory and legislative work in other jurisdictions may guide similar efforts in Canada. By presenting an overview of community paramedicine program types and training for each, this review starts to clarify the information available about community paramedicine and provides a basis for further discussion on program development, training, and education. Particularly, for the Canadian context, valuable next steps include a sub-analysis of Canadian community paramedicine programs and identifying the differences in paramedicine competencies, training, and regulations between Canadian programs and those globally.

\section{Strengths and limitations}

Limitations include that not all community paramedicine programs were captured, although the review did cover grey literature. Not all community paramedicine programs are described in the published and grey literatures, and resource limitations prevented more extensive grey literature searching (e.g., running a full Google search using a defined search strategy instead of the targeted Google search of community paramedicine program names and identifiers). Study heterogeneity prevented the meta-analysis of the top three health outcomes (i.e., 911 calls, transport to ED, hospitalization). For example, community paramedicine programs that measured the same health outcomes had vastly different population groups or were protocol only. Comparing community paramedicine programs and training was further challenged by absent or inconsistent reporting.
Fewer than half of the studies captured had a defined study design, and the other studies described only the program services and population, and generally without outcome measures. Varied data reporting and the limited ability to pool and compare studies may reflect the current state of community paramedicine. The field is growing and evolving to diverse community needs, as evidenced by the breadth of community paramedicine programs captured. Community paramedicine programs also have differing capacities to measure and report program descriptors and performance.

In the present study, the kappa coefficients suggested only moderate inter-rater reliability $(\kappa=0.41-0.60) .{ }^{83}$ As $\kappa$ is influenced by event prevalence, ${ }^{84}$ the low $\kappa$ observed could have been caused by low study inclusion during screening. Piloting screening and extraction forms, plus resolving discrepancies through discussion, supported inter-rater reliability. However, despite these limitations, this review is a comprehensive and up-to-date reflection of community paramedicine programs and reported training.

\section{CONCLUSION}

The systematic review identified 58 unique community paramedicine programs with a wide range of target populations and services. Community paramedicine training, although poorly reported, was equally diverse and included a variety of skills that were unique from the traditional paramedicine role. The highly collaborative nature of community paramedicine may warrant more training on related skills such as communication, leadership, and teamwork. Effective implementation and growth of community paramedicine may also be aided by clearer definitions of the community paramedicine role. Furthermore, enabling community paramedicine programs to gather and disseminate evidence on training and program outcomes may better inform community paramedicine education frameworks and support program growth.

Supplementary material: The supplementary material for this article can be found at https://doi.org/10.1017/cem.2019.14

Acknowledgements: We would like to acknowledge the several librarians at McMaster University and St. Michael's Hospital who provided guidance on the systematic review search strategy.

Financial support: This work was supported by a graduate studies research stipend through the Master of Public Health Program at McMaster University. Otherwise, this research received no 
specific grant or award from any funding agency, commercial, or not-for-profit sector.

Competing interests: None declared.

\section{REFERENCES}

1. Jensen JL, Marshall EG, Carter AJE, et al. Impact of a novel collaborative long-term care - EMS model: a before-andafter cohort analysis of an extended care paramedic program. Prebosp Emerg Care 2016;20(1):111-6.

2. Abrashkin KA, Washko J, Margolis A, et al. Eyes and ears on the homebound patient during an emergency response: adding video technology to a community paramedicine program. 7 Am Geriatr Soc 2015;63:S139.

3. Everden P, Eardley M, Lorgelly P, Howe A. Emergency care. Change of pace. Health Serv 7 2003;113(5865):28-30.

4. Iezzoni LI, Dorner SC, Ajayi T. Community paramedicine addressing questions as programs expand. $N$ Engl $7 \mathrm{Med}$ 2016;374(12):1107-9.

5. Pang $P$, Liao M, Litzau M, et al. Community paramedicine: a systematic review. PROSPERO International prospective register of systematic reviews. 2016. Available at: https:// www.crd.york.ac.uk/prospero/display_record.php?Recor$\mathrm{dID}=52543$ (accessed January 7, 2017).

6. Agarwal G, McDonough B, Angeles R, et al. Rationale and methods of a multicentre randomised controlled trial of the effectiveness of a Community Health Assessment Programme with Emergency Medical Services (CHAP-EMS) implemented on residents aged 55 years and older in subsidised seniors' housing buildings in Ontario, Canada. BMF Open 2015;5(6):e008110.

7. Brice JH, Overby BA, Hawkins ER, Fihe EL. Determination of infant-safe homes in a community injury prevention program. Prehosp Emerg Care 2006;10(3):397-402.

8. Smeby Jr L . Fire and Emergency Services Administration: management and leadership practices. 2nd ed. USA: Jones \& Bartlett; 2013.

9. Ontario Health Professions Regulatory Advisory Council. Paramedicine in Ontario: consideration of the Application for the Regulation of Paramedics under the Regulated Health Professions Act, 1991. 2012. Available at: https://www.hprac. org/en/resources/HPRAC_Paramedic_Report_Volume_2. pdf (accessed December 2, 2018).

10. Province of Nova Scotia. College of Paramedics Effective April 1, 2017. Available at: https://novascotia.ca/news/ release/?id=20170330003 (accessed December 2, 2018).

11. Canadian Standards Association C. Community paramedicine: framework for program development. 2017. Available at: http://cradpdf.drdc-rddc.gc.ca/PDFS/unc292/p806005_ A1b.pdf (accessed August 2018).

12. Emergency Medical Services Chiefs of Canada. The Future of EMS in Canda: defining the new road ahead. 2006. Available at: http://www.emscc.ca/docs/EMS-StrategyDocument.pdf (accessed January 12, 2019).

13. Canadian Organization of Paramedic Regulators. Vision and role statement. 2016. Available at: http://www.copr.ca/index. php/about-us/strategic-plan/vision-role-statement (accessed January 5, 2019).

14. Bigham BL, Kennedy SM, Drennan I, Morrison LJ. Expanding paramedic scope of practice in the community: a systematic review of the literature. Prehosp Emerg Care 2013;17 (3):361-72.

15. Choi BY, Blumberg C, Williams K. Mobile integrated health care and community paramedicine: an emerging emergency medical services concept. Ann Emerg Med 2016;67(3):361-6.

16. Chan J, Agarwal G. Key elements and training required among existing community paramedicine programs. 2017. Available at: https://www.crd.york.ac.uk/prospero/display_ record.php? RecordID=51774 (accessed August 30, 2017).

17. Arksey H, O'Malley L. Scoping studies: towards a methodological framework. Int 7 Soc Res Methodol 2005;8(1):19-32.

18. The Joanna Briggs Institute. Joanna Briggs Institute reviewers' manual 2015: methodology for JBI scoping reviews. 2015. Available at: http://joannabriggs.org/assets/ docs/sumari/Reviewers-Manual_Methodology-for-JBI-ScopingReviews_2015_v2.pdf (accessed December 5, 2018).

19. Pluye P, Robert E, Cargo M, et al. Mixed methods appraisal tool (MMAT) - version 2011. 2011. Available at: http://mixedmethodsappraisaltoolpublic.pbworks.com/w/page/127425845/ Download\%20the\%20MMAT (accessed December 2016).

20. Abrashkin KA, Washko J, Zhang J, et al. Providing acute care at home: community paramedics enhance an advanced illness management program-preliminary data. $7 \mathrm{Am}$ Geriatr Soc 2016;64(12):2572-6.

21. New program set to intervene to prevent readmissions, repeat ED visits due to acute exacerbations of asthma. ED Manag 2013;25(12):139-41.

22. Community paramedics fill gaps, take load off EDs. ED Manag 2014;26(3):30-4.

23. Partnership aims to cut unnecessary ED use. Hosp Case Manag 2014;22(3):29-30.

24. Mobile integrated healthcare and community paramedicine (MIH-CP): a national survey. EMS World 2015;Supp:5-16.

25. Mobile teams fill the gap between the hospital and the community. Hosp Case Manag 2016;24(2):25-6.

26. Agarwal G, Angeles R, Mcdonough B, et al. Effectiveness of a community health and wellness pilot in a subsidized seniors' apartment building: CHAP-EMS. Can 7 Diabetes 2014;38: S72.

27. Andrew J. Extending care - meeting the needs of modern healthcare. Australas 7 Prehosp Care 2011;10(2):36-7.

28. Carter A, Arab M, Harrison M. Paramedics providing palliative care at home in Nova Scotia and PEI, Canada. 7 Pain Symptom Manag 2016;52(6):e100.

29. Arendts G, Sim M, Johnston S, Brightwell R. ParaMED home: a protocol for a randomised controlled trial of paramedic assessment and referral to access medical care at home. BMC Emerg Med 2011;11:7.

30. Ashcraft SJ, Post L, Young C. Closing the gap: integrating community paramedics in stroke patients transition to home. Stroke 2017;48:AWP390.

31. Ashton C, Duffie D, Millar J. Conserving quality of life through community paramedics. Healthc Q 2017;20(2):48-53. 
32. Bennett KJ, Yuen MW M. Community paramedicine applied in a rural community. 7 Rural Health 2018;34 Suppl 1:s39-47.

33. Blumberg C. Overview of REMSA CHP with preliminary outcomes. 2014. Available at: http://mihpresources.com/programs/preliminary-outcomes-executive-summary/ (accessed January 20, 2015).

34. Boykin A, Wright D, Stevens L, Gardner L. Interprofessional care collaboration for patients with heart failure. $\mathrm{Am}$ 7 Health Syst Pharm 2018;75(1):e45-49.

35. Brice JH, Kingdon D, Runyan C. "Welcome to the world": process evaluation of a paramedic home safety intervention. Prehosp Emerg Care 2009;13(2):228-36.

36. Brown DR, Carter A, Goldstein J, et al. Evaluating the impact of a novel mobile care team (MCT) on the prevalence of ambulatory care sensitive conditions presenting to emergency medical services in Nova Scotia. C7EM 2016;18(S1): s83.

37. Chellappa DK, Munjal KG, Jervis R, et al. Evaluation of physician and paramedic perspectives on the Mount Sinai community paramedicine program. $7 \mathrm{Am}$ Geriatr Soc 2017;65:s1.

38. Cooper S, Barrett B, Black S, et al. The emerging role of the emergency care practitioner. Emerg Med 7 2004;21(5):614-8.

39. Crockett BM, Jasiak KD, Walroth TA, et al. Pharmacist involvement in a community paramedicine team. 7 Pharm Pract 2017;30(2):223-8.

40. Dixon S, Mason S, Knowles E, et al. Is it cost effective to introduce paramedic practitioners for older people to the ambulance service? Results of a cluster randomised controlled trial. Emerg Med 7 2009;26(6):446-51.

41. Drennan IR, Dainty KN, Hoogeveen P, et al. Expanding paramedicine in the community (EPIC): study protocol for a randomized controlled trial. Trials [Electronic Resource] 2014;15:473.

42. Faddy SC, McLaughlin KJ, Cox PT, Muthuswamy SS. The mental health acute assessment team: a collaborative approach to treating mental health patients in the community. Australas Psychaitry 2017;25(3):262-5.

43. Gerson LW, Schelble DT, Wilson JE. Using paramedics to identify at-risk elderly. Ann Emerg Med 1992;21(6):688-91.

44. Gray JT, Walker A. AMPDS categories: are they an appropriate method to select cases for extended role ambulance practitioners? Emerg Med 7 2008;25(9):601-3.

45. Hauswald M, Raynovich W, Brainard AH. Expanded emergency medical services: the failure of an experimental community health program. Prehosp Emerg Care 2005;9(2):250-3.

46. Jensen JL, Travers AH, Bardua DJ, et al. Transport outcomes and dispatch determinants in a paramedic long-term care program: a pilot study. CFEM 2013;15(4):206-13.

47. Kusel E, Savino PB. Boots on the ground. Alameda County, Calif., community paramedics curb hospital readmissions \& non-emergent 9-1-1 use. 7 Emerg Med Serv 2015;40 (12):55-7.

48. Marshall EG, Clarke B, Peddle S, Jensen J. Care by design: new model of coordinated on-site primary and acute care in long-term care facilities. Can Fam Physician 2015;61(3): e129-34.
49. Martin-Misener R, Downe-Wamboldt B, Cain E, Girouard M. Cost effectiveness and outcomes of a nurse practitioner-paramedic-family physician model of care: the Long and Brier Islands study. Prim Health Care Res 2009;10 (1):4-25.

50. Mason S, Wardrope J, Perrin J. Developing a community paramedic practitioner intermediate care support scheme for older people with minor conditions. Emerg Med 7 2003;20(2):196-8.

51. Mason S, Knowles E, Freeman J, Snooks H. Safety of paramedics with extended skills. Acad Emerg Med 2008;15 (7):607-12.

52. Mason S, Knowles E, Colwell B, et al. Effectiveness of paramedic practitioners in attending 999 calls from elderly people in the community: cluster randomised controlled trial. $B M 7$ 2007;335(7626):919.

53. McCarthy J, Dalgarno D. Care in the community-home transfusion by community paramedics. Transfusion 2016;56:226A.

54. McCarthy P, Brown A, Nystrom P, Ho J. Impact of community paramedic program on health service utilization. 2017. Available at: https://www.eventscribe.com/2017/SAEM/ ajaxcalls/PresentationInfo.asp? efp=SFFWWlhCWFYzMzA1\&PresentationID=267683\&rnd=0.6724276 (accessed October 18, 2018).

55. MedStar Mobile Healthcare M. 2015. Available at: http:// www.medstar911.org/Websites/medstar911/files/Content/ 1089414/MedStar_CHF_Program_Overview_-_2015.pdf (accessed October 18, 2016).

56. National Association of Emergency Medical Technicians, NAEMT. Mobile integrated healthcare and community paramedicine (MIH-CP): a national survey. 2015. Available at: https://www.naemt.org/docs/default-source/communityparamedicine/naemt-mih-cp-report.pdf?sfvrsn=4 (accessed October 18, 2016).

57. Nejtek VA, Aryal S, Talari D, et al. A pilot mobile integrated healthcare program for frequent utilizers of emergency department services. Am 7 Emerg Med 2017;35(11):1702-5.

58. Patterson DG, Coulthard C, Garberson LA, et al. What is the potential of community paramedicine to fill rural health care gaps? 7 Health Care Poor Underserved 2016;27(4A):144-58.

59. Roeper B, Mocko J, O'Connor LM, et al. Mobile integrated healthcare intervention and impact analysis with a medicare advantage population. Popul Health Manag 2018;21(5):34956.

60. Shah MN, Caprio TV, Swanson P, et al. A novel emergency medical services-based program to identify and assist older adults in a rural community. 7 Am Geriatr Soc 2010;58 (11):2205-11.

61. Snooks HA, Anthony R, Chatters R, et al. Paramedic assessment of older adults after falls, including community care referral pathway: cluster randomized trial. Ann Emerg Med 2017;70(4):495-505.

62. Snooks HA, Anthony R, Chatters R, et al. Support and assessment for fall emergency referrals (SAFER) 2: a cluster randomised trial and systematic review of clinical effectiveness and cost-effectiveness of new protocols for emergency ambulance paramedics to assess older people following a fall with referral 
to community-based care when appropriate. Health Technol Assess 2017;21(13):1-218.

63. Snooks H, Kearsley N, Dale J, et al. Towards primary care for non-serious 999 callers: results of a controlled study of "Treat and Refer" protocols for ambulance crews. Qual Saf Health Care 2004;13(6):435-43.

64. Snooks H, Anthony R, Chatters R, et al. Support and assessment for fall emergency referrals (SAFER 2) research protocol: cluster randomised trial of the clinical and cost effectiveness of new protocols for emergency ambulance paramedics to assess and refer to appropriate communitybased care. BM7 Open 2012;2:e002169.

65. Swain AH, Hoyle SR, Long AW. The changing face of prehospital care in New Zealand: the role of extended care paramedics. N Z Med F 2010;123(1309):11-4.

66. Tangherlini N, Villar J, Brown J, et al. The HOME team: evaluating the effect of an EMS-based outreach team to decrease the frequency of 911 use among high utilizers of EMS. Prehosp Disaster Med 2016;31(6):603-7.

67. The California Health Care Foundation, CHCF. Overview: California's community paramedicine pilot projects. 2017. Available at: https://www.chcf.org/wp-content/uploads/ 2018/05/CommunityParamedicinePilotProjects.pdf (accessed January 2, 2018).

68. Wilcox MR. Community paramedicine in a rural setting. Minnesota's approach includes free clinics and a mobile unit that travels the community. EMS World 2016;45(2):17-9.

69. Yaworski H, Hutlet P, Grierson R, et al. HIV point of care testing by community paramedics in a vulnerable population: a pilot study. CFEM 2016;18(S1):s126.

70. Agarwal G, Angeles R, Pirrie M, et al. Effectiveness of a community paramedic-led health assessment and education initiative in a seniors' residence building: the Community Health Assessment Program through Emergency Medical Services (CHAP-EMS). BMC Emerg Med 2017;17(1):8.

71. Agarwal G, McDonough B, McLeod B, et al. A community health and wellness pilot in a subsidised seniors' apartment building: CHAP-EMS. Can 7 Cardiol 2013;29(10 Suppl 1):S327-8.

72. Agarwal G, Mcdonough B, Mcleod B, et al. A community health awareness program in subsidized seniors homes: using CANRISK as a risk assessment and health promotion tool. Can 7 Diabetes 2013;37:S75.

73. Agarwal G, Angeles RN, McDonough B, et al. Development of a community health and wellness pilot in a subsidised seniors' apartment building in Hamilton, Ontario: Community Health Awareness Program delivered by Emergency Medical Services (CHAP-EMS). BMC Res Notes 2015;8:113.

74. New program set to intervene to prevent readmissions, repeat ED visits due to acute exacerbations of asthma. ED Manag 2013;25(12):139-41.

75. Misner D. Community paramedicine: part of an integrated healthcare system. Emerg Med Serv 2005;34(4):89-90.

76. Zavadsky $M$. Trained paramedics provide ongoing support to frequent 911 callers, reducing use of ambulance and emergency department services. 2016. Available at: https:// innovations.ahrq.gov/profiles/trained-paramedics-provideongoing-support-frequent-911-callers-reducing-use-ambulanceand (accessed October 2016).

77. MedStar Mobile Healthcare M. Program overview - high utilizer 9-1-1/emergency department patients. 2017. Available at: https://www.naemt.org/Files/communityparamedicinegrid/CHPProgramOverview.pdf (accessed January 2, 2018).

78. Harrison M. Paramedics providing palliative care at home. 2017. Available at: https://novascotia.ca/dhw/ehs/palliativecare.asp (accessed February 2019).

79. LaCalle E, Rabin E. Frequent users of emergency departments: the myths, the data, and the policy implications. Ann Emerg Med 2010;56(1):42-8.

80. Brault I, Kilpatrick K, D'Amour D, et al. Role clarification processes for better integration of nurse practitioners into primary healthcare teams: a multiple-case study. Nurs Res Pract 2014;2014:170514.

81. Minnesota Department of Health. Community paramedics (CP). 2017. Available at: http://www.health.state.mn.us/ divs/orhpc/workforce/emerging/cp/ (accessed October 18, 2018).

82. National Association of State EMS Officials CPMIH Committee. State by state community paramedicine - Mobile Integrated Healthcare (CP-MIH) status board. 2018. Available at: https://nasemso.org/wp-content/ uploads/CP_State_Status_Board_CURRENT.pdf (accessed October 2018).

83. McHugh ML. Interrater reliability: the kappa statistic. Biochem Med (Zagreb) 2012;22(3):276-82.

84. Mandrekar JN. Measures of interrater agreement. 7 Thorac Oncol 2011;6(1):6-7. 